\title{
Atypical proliferative nodule in congenital melanocytic nevus with dural invasion: a case report
}

\author{
Jae Hoon Jung ${ }^{1 \star}$, \\ Kee-Taek Jang ${ }^{2 \star}$, \\ Ara Kim ${ }^{1}$, \\ So Young $\operatorname{Lim}^{1}$ \\ Departments of ${ }^{1}$ Plastic Surgery and \\ ${ }^{2}$ Pathology, Samsung Medical Center, \\ Sungkyunkwan University College of \\ Medicine, Seoul, Korea
}

Here we report a case of a focal atypical proliferative nodule (PN) arising from a congenital melanocytic nevus (CMN). Diagnosis was challenging because it had both benign and malignant clinical features. Unusual histopathology, immunohistochemistry, and intraoperative findings of this atypical PN are discussed. A 5-year-old girl was admitted for a congenital $5 \times 5 \mathrm{~cm}$ sized scalp mass. This hemangioma-like soft mass showed biphasic characteristics such as a slow, gradual, and benign increase in size but worrisome dural invasion with cranial bone defect. We removed the scalp mass with clear resection margins. Interoperatively, we found that the cranial bone defect had already filled. Histopathologic examination showed CMN with focal atypical PN. The nodule showed sharp demarcation and cellular pleomorphism. However, in immunohistochemical study, Ki-67 proliferation index and expression levels of protein S-100 and Melan-A were very low. These were unusual findings of atypical PNs. Despite her worrisome preoperative radiologic features, she showed an indolent clinical course compatible with previously reported biologic behavior. The patient underwent follow-up inspection with magnetic resonance imaging every 6 months for up to 3 years. The nodule appeared to be stationary at the last visit.

Keywords: Dura mater / Melanocytic nevus syndrome, congenital / Neoplasm invasiveness / Tissue expansion devices

\section{INTRODUCTION}

Congenital melanocytic nevus (CMN) is associated with secondary melanocytic tumors such as benign proliferative nodules (PNs) and malignant melanomas. Atypical PNs can also be found in CMN histologically. They are difficult to differentiate from malignant melanomas due to their nearly identical morphology [1]. While PNs generally show a benign clinical course as previously reported, their atypical histopathologic features with long-term follow up are not well-known. The biological potential of atypical PNs remains uncertain. When clinical and

Correspondence: So Young Lim

Department of Plastic Surgery, Samsung Medical Center, Sungkyunkwan

University School of Medicine, 81 Irwon-ro, Gangnam-gu, Seoul 06351, Korea

E-mail: pslisy@naver.com

*The first two authors contributed equally to this work.

Received February 5, 2019 / Revised March 13, 2019 / Accepted March 26, 2019 histopathologic findings are ambiguous, it becomes even more challenging to decide if the possible malignancy of large CMNs warrants radical excision. It is thus imperative to rule out malignancy to choose appropriate treatments.

We report a pediatric case of a large scalp CMN showing a focal atypical PN. The CMN was locally destructive with dural invasion and cranial bone defect, making it difficult to decide whether the atypical PN on CMN was benign or malignant. On immunohistochemical workup, this case showed unusual features of decreased S-100, Melan-A, and Ki-67 expression. Unusual histopathology, immunohistochemistry, and intraoperative findings of this atypical PN are also discussed.

\section{CASE REPORT}

A 6-year-old girl presented at an outpatient clinic with a scalp 
mass that had been present since her birth. She had initially visited the Samsung Medical Center at the age of 1 month with a $1.5 \times 1.5 \mathrm{~cm}$ hemangioma-like scalp mass at a vertex. There was no medical, familial, or psychosocial history relevant to this mass. This soft-tissue mass was purple in color, hard, and fixed to the scalp on initial palpation. It had shown a gradual increase in size proportional to her growth. However, a cobblestone appearance with protrusion became prominent during clinical observation (Fig. 1). We could not exclude possible malignancy. We considered it risky to delay intervention until adulthood. Thus, we planned tissue expander placement prior to surgical excision because the base of the mass was too large for primary closure. We preoperatively conducted computed tomography (CT), magnetic resonance imaging (MRI), and positron-emission tomography (PET) imaging studies to check the extent of the tumor and evaluate its malignancy. Radiologic CT and MRI findings showed a $5-\mathrm{cm}$ hypervascular mass with intradural extension and parieto-occipital bone defect which was an unfavorable finding that required further differential diagnosis for hemangioma, vascular malformation, and hypervascular solid mass (Figs. 2, 3). PET imaging showed no evidence of metastasis. To maintain her physiological cranial suture lines, we delayed inserting the tissue expander until she became 3 years old

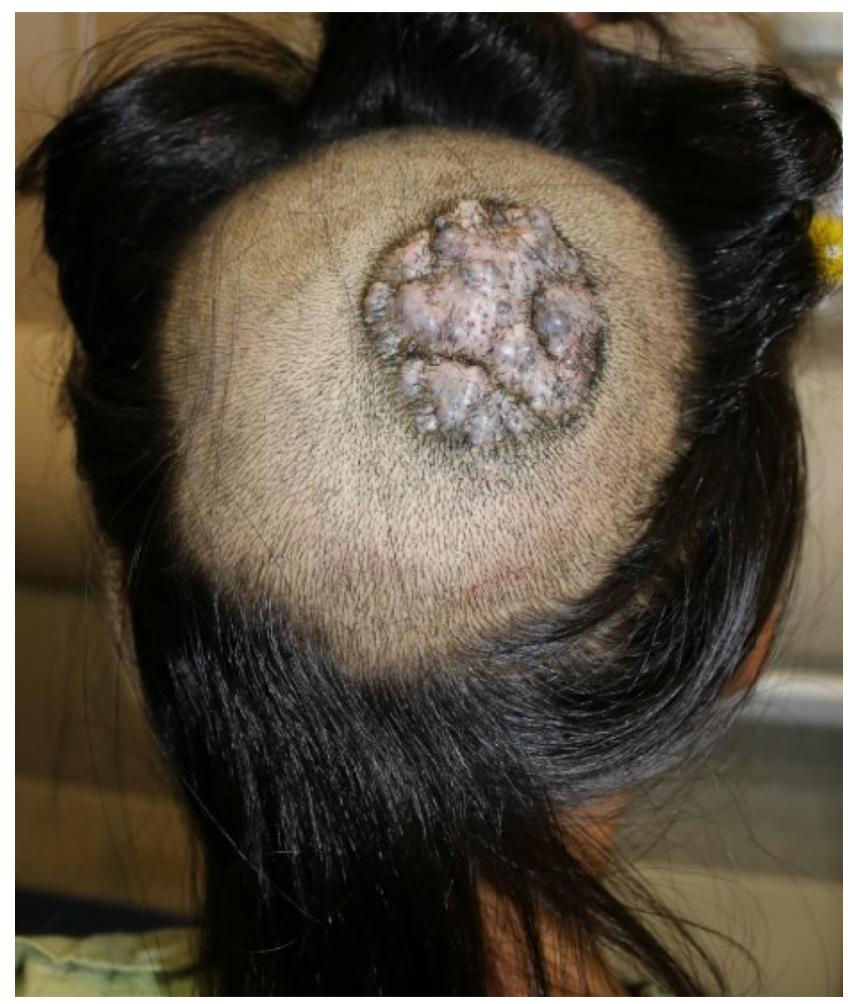

Fig. 1. Preoperative photograph of a mass in a 5-year-old patient. A protruding cobblestone-like scalp mass was $5 \times 5 \mathrm{~cm}$ in size immediately before tissue expander insertion. under close observation. When the patient became 5 years old, we inserted the expander and scheduled a total mass excision (Fig. 4). The expander inserted was textured croissant type tissue expander (Groupe Sebbin SAS, Boissy-l'Aillerie, France; $50 \times 110 \times 45 \mathrm{~mm}, 120 \mathrm{~mL}$ ). At the outpatient clinic, we injected 10-20 mL normal saline to the expander once a week. Eventu-

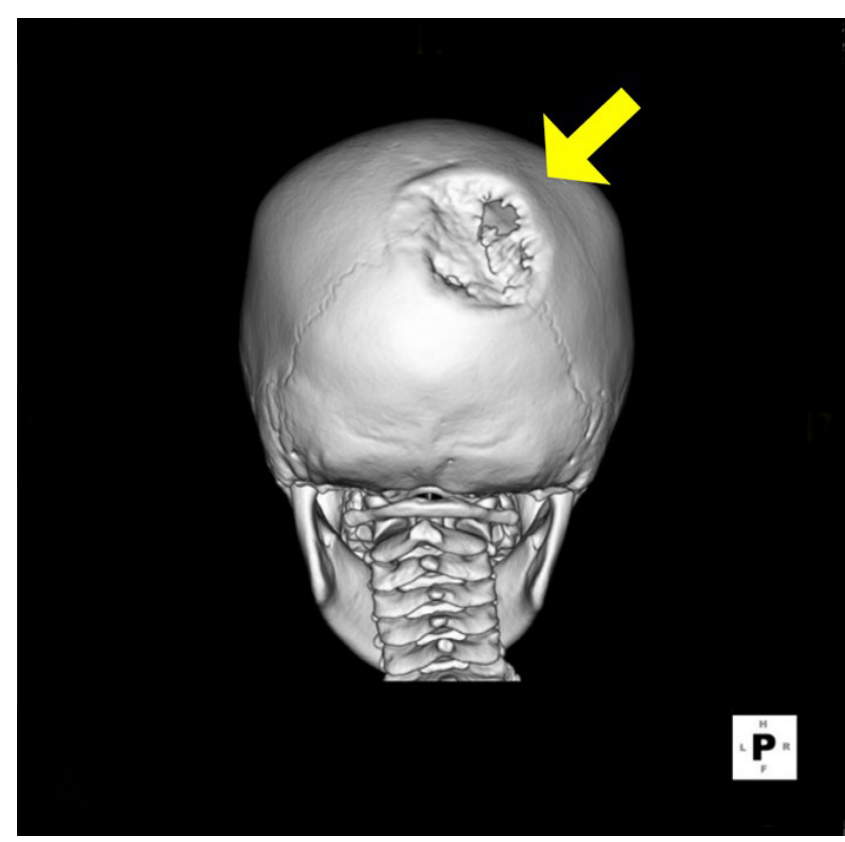

Fig. 2. Preoperative computed tomography (CT) image. A yellow arrow indicates bone defect on preoperative CT image.

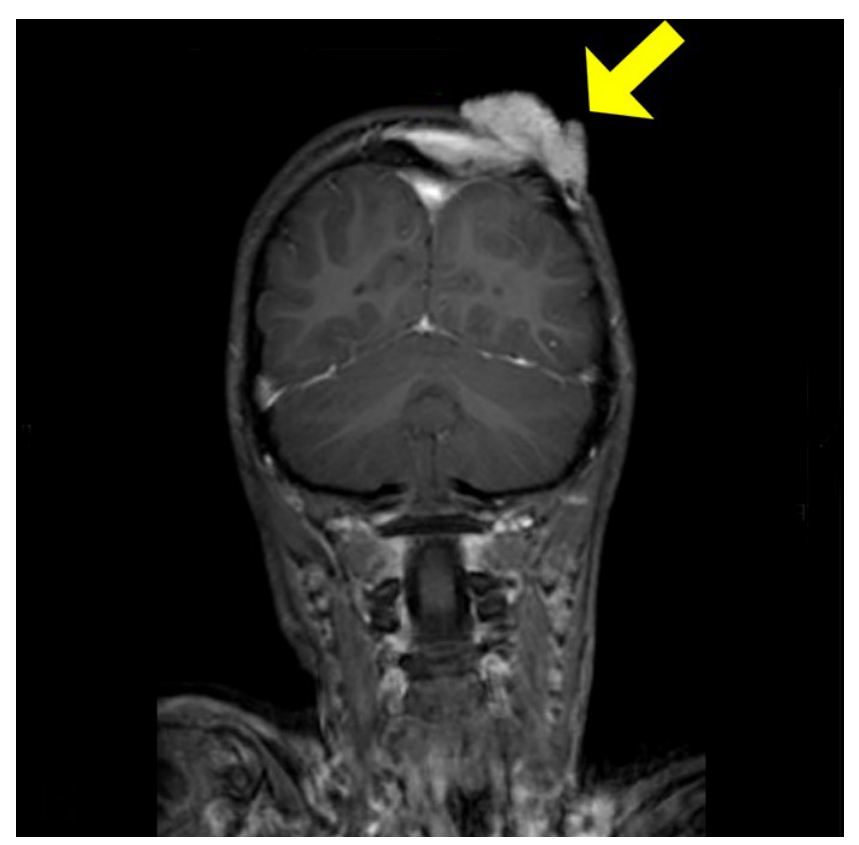

Fig. 3. Preoperative magnetic resonance (MR) image. A yellow arrow indicates scalp tumor with hypervascular feature of dural extension on preoperative MR image. 

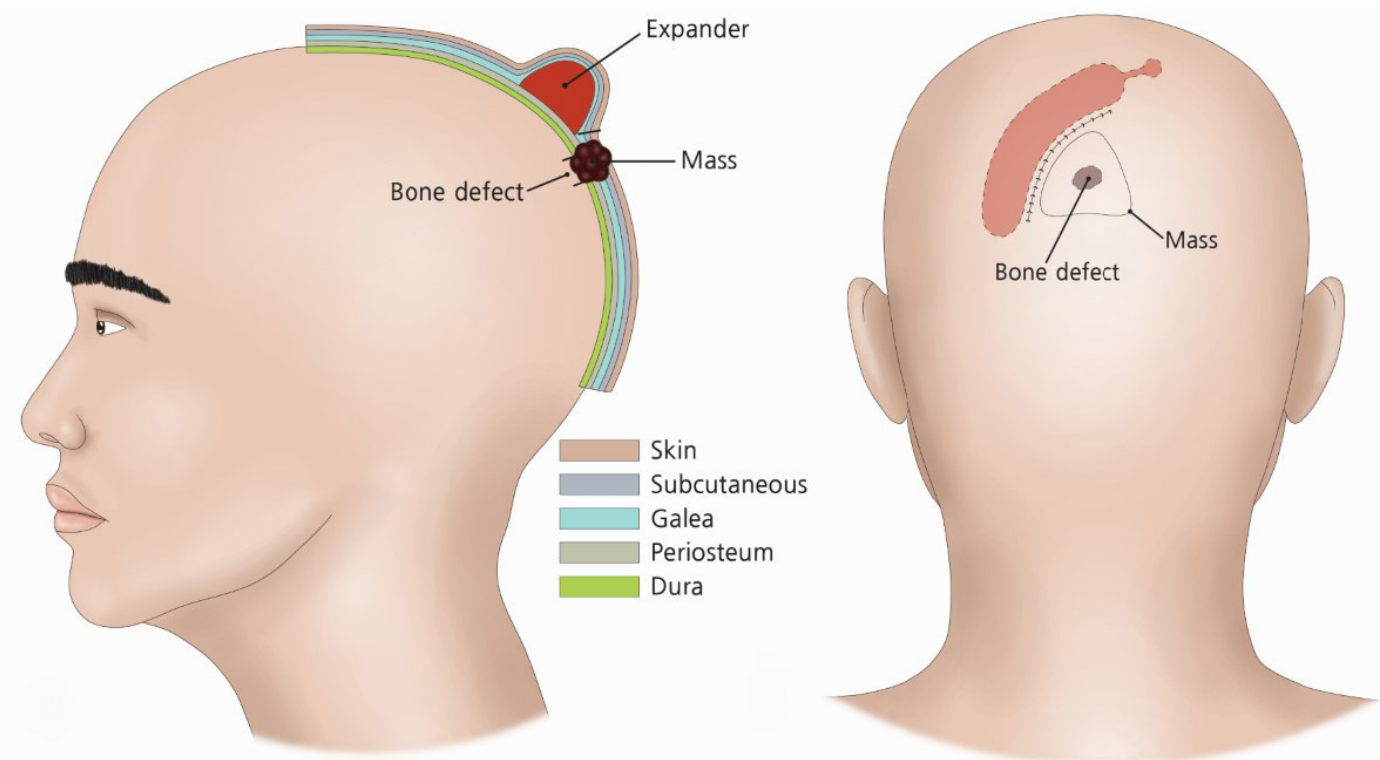

Fig. 4. Preoperative diagrams of the expander removal. Both lateral and posterior views show the location of expander insertion. The expander was inserted between galeal and periosteal layers.

ally, the expander was fully extended to $160 \mathrm{~mL}$ after 3 months (from January 2016 to March 2016). We removed it and completely excised the mass with defect coverage by advancement scalp flap. During the operation, we observed that the parietooccipital bone defect beneath the mass had already filled with new cranial bone (Fig. 5). After confirming clear resection margins on frozen examination, we finished the operation.

Pathological examination of the resected specimen showed a large $(6.7 \times 5.2 \mathrm{~cm})$ scalp mass with cobblestone appearance. Histopathologic examination revealed a large $\mathrm{CMN}$ with a focal atypical cell nodule. The CMN measured $4.5 \times 4.2 \mathrm{~cm}$ and the nodule measured $0.5 \mathrm{~cm}$. The nodule showed sharp demarcation from the surrounding nevus. It consisted of epithelioid cells with pleomorphic nuclei and abundant eosinophilic cytoplasm (Fig. 6). However, there was no mitosis, epidermal effacement, or necrosis. Immunohistochemical staining of the nodule showed decreased S-100 and Melan-A expression with a very low Ki-67 proliferation index. Based on histology and immunostaining results, we diagnosed this mass as an atypical $\mathrm{PN}$ arising from $\mathrm{CMN}$.

There was no gross evidence of recurrent mass growth at postoperative 4 months. On postoperative 6-month follow-up MRI, vivid enhancement beneath the resected tumor region showed evidence of spontaneous parieto-occipital bone healing, consistent with our intraoperative finding (Fig. 7). We also noted a residual tumor on the dural layer. With palpation, the previous bone defect seemed to have healed remarkably. Postoperative 1-year follow-up MRI and CT revealed no significant

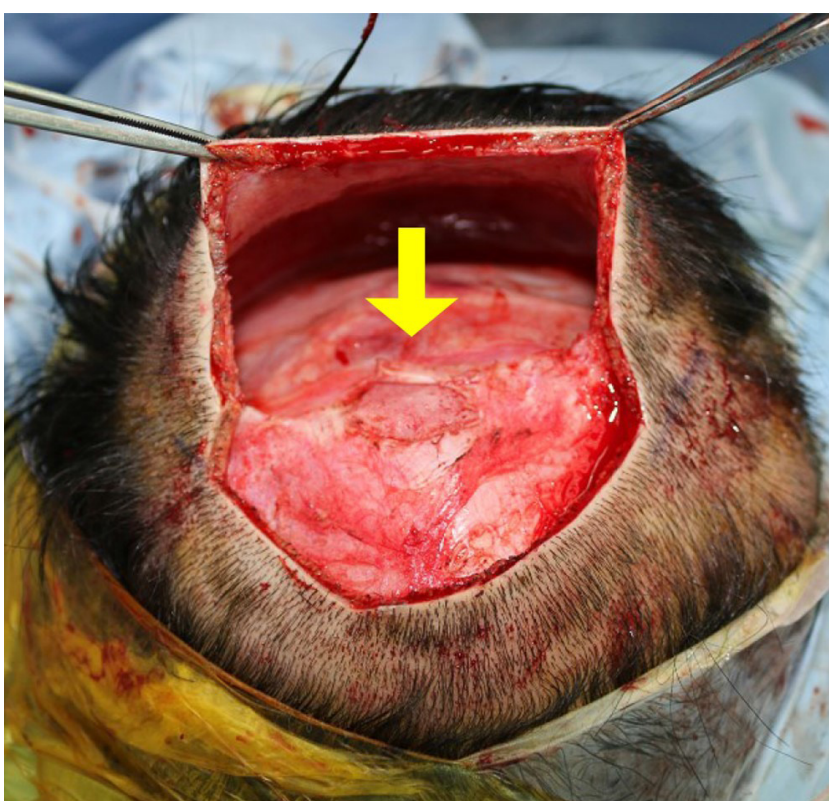

Fig. 5. Intraoperative photograph. A yellow arrow indicates complete bone healing of previous cranium defect.

interval change in the residual mass or the bony defect. The patient reported no palpable mass or symptoms. Our management strategy was 6 months of outpatient follow-up for at least 3 years.

\section{DISCUSSION}

Therapeutic excision of large CMNs is controversial regarding 

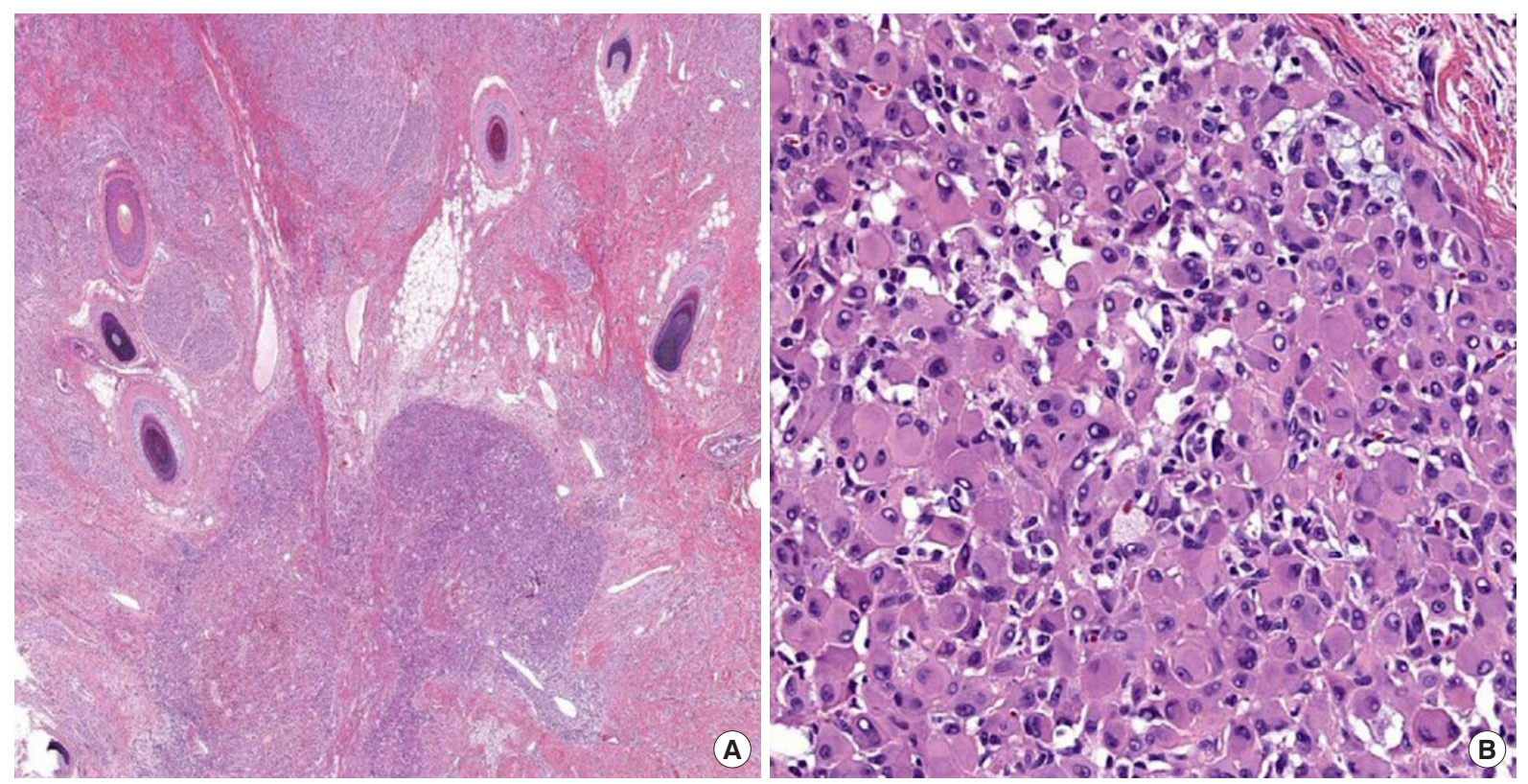

Fig. 6. Histopathological features. (A) Sharp demarcation was found in a focal atypical cell nodule of a congenital melanocytic nevus (H\&E, $\times 40)$. (B) Cellular pleomorphism was found in a focal atypical cell nodule of a congenital melanocytic nevus (H\&E, $\times 200)$.

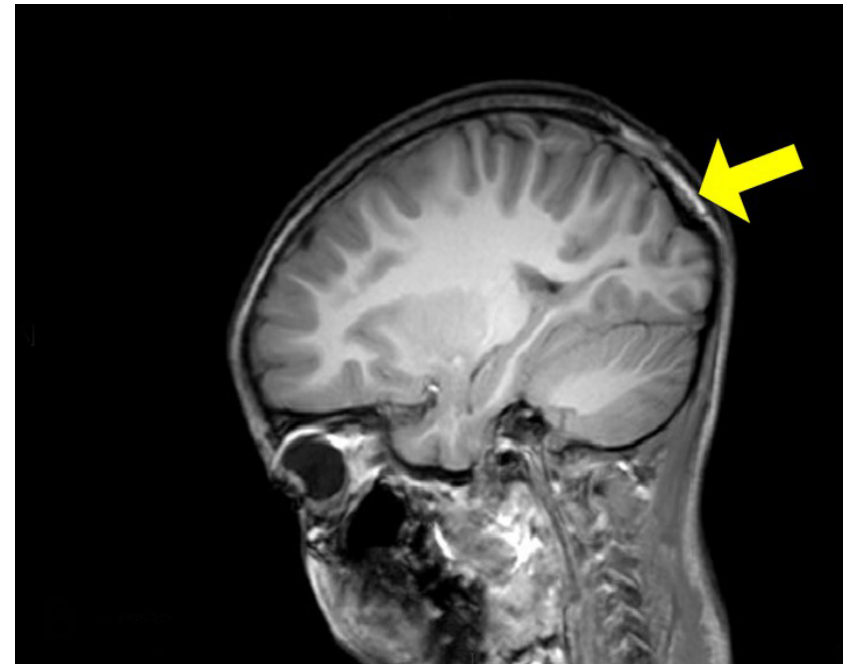

Fig. 7. Postoperative 6-month follow-up magnetic resonance image. A yellow arrow indicates spontaneous healing of the parieto-occipital bone.

risks and benefits associated with low risk melanoma versus reconstruction scars and graft contours. Therefore, differential diagnosis is important for determining the risk of malignancy [2]. $\mathrm{PN}$ is one feature of CMNs, with an incidence of up to $2 \%$ in newborns with CMN. There is a lack of data on atypical features of PN. Its diagnosis and appropriate treatment planning have been uncertain [3]. Atypical PNs that arise from CMN are difficult to histologically differentiate from malignant melanoma. Sometimes they have nearly identical pathologic and clinical features to melanoma. Treatment for them has little consen- sus [3]. Currently, the only reliable criterion of malignant melanoma is metastatic spread [1].

We introduced immunohistochemical study of atypical PN. Such study has been rarely considered in previous studies to exclude malignant melanoma. One of interesting histopathologic findings of the atypical PN in the present case was decreased expression of melanocytic markers S-100 protein and Melan-A in accordance with very low proliferation index in Ki-67 immunostaining. Such pattern of decreased S-100 protein and Melan-A expression can cause confusion in differential diagnosis of melanocytic tumors because these markers are relatively sensitive to melanocyte-originated lesions ranging from benign nevus to melanoma. Although we did observe decreased expression of S-100 protein and Melan-A, the Ki-67 proliferation index and mitotic count were very low in this case, suggesting clinically indolent benign behavior.

Clinical features played critical roles in the final diagnosis and management plan for this case. It was difficult to confirm the need for urgent excision based on contradictory clinical features of this scalp mass. It appeared to be benign given its slow gradual growth proportional to her normal head growth. However, malignancy was suspected based on worrisome image findings of cranial invasion with grossly protruding nodular appearance. However, the cranial bone defect ultimately healed spontaneously. Thus, dural invasion and cranial bone defect should not be considered as signs of malignancy, especially in pediatric patients with CMN with or without PN. Previous publications have indicated that atypical PNs in CMNs are usu- 
ally benign. However, lesions reported in previous studies only showed limited features such as ulceration or erosion whereas none showed local tissue invasion or metastasis known to be a reliable malignancy indicator [4-8]. Biopsy may be reasonable if there is any sign of local recurrence. Atypical PN should not be interpreted as melanoma.

Tissue expansion has been frequently used as an adjunctive modality for scalp reconstruction [9]. In the present case, the scalp tissue that bore the lesion seemed highly non-surgically dissected from the underlying cranium due to inflating effect of the expander. Moreover, tissue expansion might have promoted bone self-healing by elevating the scalp flap, resulting in the removal of the lesion's mass effects on the cranium. The pressure and mass effects on the cranium were released by the force pushing outward. This process might have been sufficiently slow and gradual to allow spontaneous bone healing of the cranium defect.

In summary, we report a focal atypical PN that arises from a CMN in a pediatric patient with both benign and malignant clinical features. The atypical PN in this case showed unique histopathologic findings of a low Ki-67 proliferation index with decreased expression of melanocytic markers S-100 protein and Melan-A, all of which were different from previous descriptions of atypical PN associated with CMN. Although preoperative radiologic images showed unfavorable characteristics that suggested possible malignancy, we excluded malignancy based on histopathologic and immunohistochemistry findings and clinical correlation of spontaneous cranial bone healing. Tissue expansion in this case might have affected the regression of this tumor and promoted bone formation beneath it. Further longterm follow-up is needed to detect any signs of malignancy development.

\section{NOTES}

\section{Conflict of interest}

No potential conflict of interest relevant to this article was reported.

\section{Ethical approval}

The study was approved by the Institutional Review Board of Samsung Medical Center (IRB No. SMC 2019-01-150-001) and performed in accordance with the principles of the Declaration of Helsinki. Written informed consent was obtained.

\section{Patient consent}

The patient provided written informed consent for the publication and the use of her images.

\section{ORCID}

Jae Hoon Jung https://orcid.org/0000-0002-8847-8484

Kee-Taek Jang https://orcid.org/0000-0001-7987-4437

Ara Kim

https://orcid.org/0000-0002-5670-7043

So Young Lim https://orcid.org/0000-0003-1541-9827

\section{REFERENCES}

1. Flux K, Hartschuh W. Congenital spindle cell naevus with unusual transformation: proliferative nodule or melanoma? Acta Derm Venereol 2012;92:152-5.

2. Bauer BS, Adler N. Congenital melanocytic nevi. In: Rodriguez ED, Losee JE, Neligan PC, editors. Plastic Surgery. 3rd ed. Philadelphia: Saunders; 2012. p. 837-54.

3. Phadke PA, Rakheja D, Le LP, Selim MA, Kapur P, Davis A, et al. Proliferative nodules arising within congenital melanocytic nevi: a histologic, immunohistochemical, and molecular analyses of 43 cases. Am J Surg Pathol 2011;35:656-69.

4. Herron MD, Vanderhooft SL, Smock K, Zhou H, Leachman SA, Coffin C. Proliferative nodules in congenital melanocytic nevi: a clinicopathologic and immunohistochemical analysis. Am J Surg Pathol 2004;28:1017-25.

5. Crowson AN, Magro CM, Mihm MC. The melanocytic proliferations: a comprehensive textbook of pigmented lesions. New York: Wiley-Liss; 2001.

6. Bastian BC, Xiong J, Frieden IJ, Williams ML, Chou P, Busam K, et al. Genetic changes in neoplasms arising in congenital melanocytic nevi: differences between nodular proliferations and melanomas. Am J Pathol 2002;161:1163-9.

7. Giam YC, Williams ML, Leboit PE, Orlow SJ, Eichenfield LF, Frieden IJ. Neonatal erosions and ulcerations in giant congenital melanocytic nevi. Pediatr Dermatol 1999;16:354-8.

8. de Vooght A, Vanwijck R, Gosseye S, Bayet B. Pseudo-tumoral proliferative nodule in a giant congenital naevus. Br J Plast Surg 2003;56:164-7.

9. Colonna M, Cavallini M, De Angelis A, Preis FW, Signorini M. The effects of scalp expansion on the cranial bone: a clinical, histological, and instrumental study. Ann Plast Surg 1996;36: 255-60. 